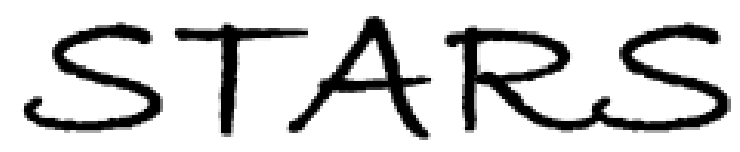

University of Central Florida

STARS

$1-1-2001$

\title{
Mathematical model for the irradiance probability density function of a laser beam propagating through turbulent media
}

\author{
M. A. Al-Habash \\ L. C. Andrews \\ University of Central Florida \\ R. L. Phillips \\ University of Central Florida
}

Find similar works at: https://stars.library.ucf.edu/facultybib2000

University of Central Florida Libraries http://library.ucf.edu

This Article is brought to you for free and open access by the Faculty Bibliography at STARS. It has been accepted for inclusion in Faculty Bibliography 2000s by an authorized administrator of STARS. For more information, please contactSTARS@ucf.edu.

\section{Recommended Citation}

Al-Habash, M. A.; Andrews, L. C.; and Phillips, R. L., "Mathematical model for the irradiance probability density function of a laser beam propagating through turbulent media" (2001). Faculty Bibliography 2000s. 2907.

https://stars.library.ucf.edu/facultybib2000/2907

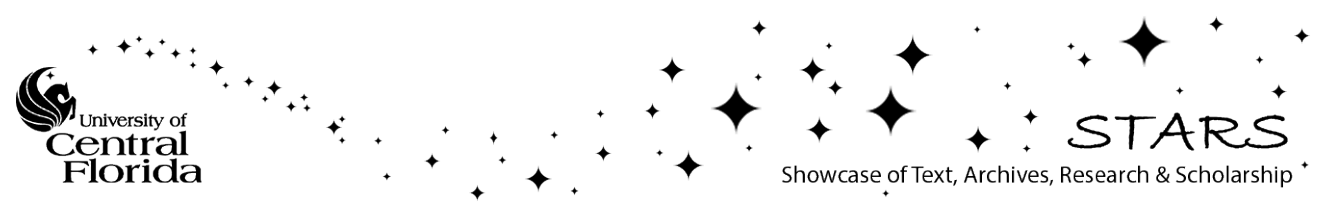




\section{Mathematical model for the irradiance probability density function of a laser beam propagating through turbulent media}

\author{
M. A. Al-Habash* \\ L. C. Andrews, MEMBER SPIE \\ University of Central Florida \\ Department of Mathematics \\ and \\ School of Optics/CREOL \\ Orlando, Florida 32816 \\ R. L. Phillips \\ Florida Space Institute \\ and \\ University of Central Florida \\ Department of Electrical and Computer \\ Engineering \\ Orlando, Florida 32816
}

\begin{abstract}
We develop a model for the probability density function (pdf) of the irradiance fluctuations of an optical wave propagating through a turbulent medium. The model is a two-parameter distribution that is based on a doubly stochastic theory of scintillation that assumes that small-scale irradiance fluctuations are modulated by large-scale irradiance fluctuations of the propagating wave, both governed by independent gamma distributions. The resulting irradiance pdf takes the form of a generalized $K$ distribution that we term the gamma-gamma distribution. The two parameters of the gamma-gamma pdf are determined using a recently published theory of scintillation, using only values of the refractive-index structure parameter $C_{n}^{2}$ (or Rytov variance) and inner scale $I_{0}$ provided with the simulation data. This enables us to directly calculate various log-irradiance moments that are necessary in the scaled plots. We make a number of comparisons with published plane wave and spherical wave simulation data over a wide range of turbulence conditions (weak to strong) that includes inner scale effects. The gamma-gamma pdf is found to generally provide a good fit to the simulation data in nearly all cases tested. (ㄷ 2001 Society of Photo-Optical Instrumentation Engineers. [DOI: 10.1117/1.1386641]
\end{abstract}

Subject terms: lasers; propagation; irradiance.

Paper 200410 received Oct. 17, 2000; revised manuscript received Feb. 12, 2001; accepted for publication Feb. 27, 2001.

\section{Introduction}

For more than three decades the scientific community has expressed interest in the possibility of using high-data-rate optical transmitters for radar and optical communications. This interest stems from the advantages offered by optical wave systems over conventional rf systems such as smaller antenna: less mass, power, and volume; and the intrinsic narrow-beam and high-gain nature of lasers. Applications that could benefit from laser communication, or lasercom, systems are those that have platforms with limited weight and space, require very high data links, and must operate in an environment where fiber optic links are not practical, such as between buildings across cities or supporting military tactical operations. Also, there has been a lot of interest over the years in the possibility of using optical transmitters for satellite communications. Although many of the early developmental programs were terminated due to funding cutbacks, there was renewed interest during the decade of the 1990s in the use of optical transmitters for communication channels connecting ground/airborne-to-space or space-to-ground/airborne data links. ${ }^{1,2}$

The performance of a laser radar or lasercom system can be significantly diminished by turbulence-induced scintillation resulting from beam propagation through the atmosphere. Specifically, scintillation can lead to power losses at

*Current affiliation: TeraBeam Networks, 14833 NE 87th St., Bldg. C, Redmond, WA 98052. the receiver and eventually to fading of the received signal below a prescribed threshold. The reliability of an optical system operating in such an environment can be deduced from a mathematical model for the probability density function (pdf) of the randomly fading irradiance signal. For that reason, one of the goals in studying optical wave propagation through optical turbulence is the identification of a tractable pdf of the irradiance under all irradiance fluctuation conditions. In addition, it is beneficial if the free parameters of that pdf can be tied directly to atmospheric parameters.

The purpose of this paper is to develop a tractable pdf model for the irradiance fluctuations in homogeneous, isotropic turbulence that can be useful in studying the performance characteristics of various laser radar and lasercom systems that operate in an environment in which the refractive-index structure parameter $C_{n}^{2}$ and inner scale $l_{0}$ can be reasonably estimated. In an effort to validate the use of this pdf model in practical applications, we make comparisons of it with published plane wave pdf simulation data $^{3}$ and spherical wave pdf simulation data ${ }^{4,5}$ under a wide variety of simulated turbulence conditions, including inner-scale effects.

\section{Background}

Over the years, many irradiance pdf models have been proposed with varying degrees of success. Under weak irradiance fluctuations, the irradiance pdf is generally accepted to 
be log normal. Indeed, measurements of the lower order normalized irradiance moments under weak conditions generally fit the values predicted by the lognormal model. ${ }^{6,7}$ In spite of data that support the log-normal model, it has also been observed that the log-normal pdf can underestimate the peak of the probability density function and underestimate the behavior in the tails as compared with measured data. ${ }^{5,8}$ Underestimating the tails of a pdf has important consequences on radar and communication systems where detection or fade probabilities are calculated primarily over the tails of the given pdf.

As the strength of turbulence increases and multiple self-interference effects must be taken into account, greater deviations from lognormal statistics are present in measured data. ${ }^{6-8}$ Eventually, however, the radiation field of the wave can be approximated by a zero-mean Gaussian distribution, and, thus, the irradiance statistics are then governed by the negative exponential distribution. The negative exponential distribution is considered a limit distribution for the irradiance and is therefore approached only far into the saturation regime.

Some of the most useful of the current pdf models have evolved from an assumed modulation process as the wave propagates through optical turbulence.$^{5,8-15}$ One of the first such models to gain wide acceptance for a variety of applications under strong fluctuation conditions was the $K$ distribution. This distribution was originally proposed as a model for non-Rayleigh sea echo, ${ }^{9}$ but it was later discovered that it also provides excellent agreement with numerous experimental data involving radiation scattered by turbulent media under strong fluctuation conditions. ${ }^{10,11}$ Although usually formulated from a discrete point of view, the $K$ distribution can be derived from a modulation process wherein the pdf of irradiance is assumed governed by the conditional negative exponential distribution

$p_{1}(I \mid b)=\frac{1}{b} \exp (-I / b), \quad I>0$,

where the mean irradiance $b=\langle I\rangle$ is itself a random quantity. The unconditional pdf for the irradiance is obtained by calculating the expected value

$p(I)=\int_{0}^{\infty} p_{1}(I \mid b) p_{2}(b) \mathrm{d} b, \quad I>0$,

where $p_{2}(b)$ is the distribution function of the fluctuating mean irradiance, assumed to be the gamma distribution

$p_{2}(b)=\frac{\alpha(\alpha b)^{\alpha-1}}{\Gamma(\alpha)} \exp (-\alpha b), \quad b>0, \quad \alpha>0$.

In Eq. (3), $\Gamma(\alpha)$ is the gamma function and $\alpha$ is a positive parameter related to the effective number of discrete scatterers. The resulting distribution arising from Eq. (2) is given by

$p(I)=\frac{2 \alpha}{\Gamma(\alpha)}(\alpha I)^{(\alpha-1) / 2} K_{\alpha-1}(2 \sqrt{\alpha I}), \quad I>0, \quad \alpha>0$, where $K_{p}(x)$ is a modified Bessel function of the second kind. Because of the presence of this particular Bessel function, the pdf [Eq. (4)] is known as the $K$ distribution.

The scintillation index predicted by the $K$ distribution assumes the form $\sigma_{I}^{2}=1+2 / \alpha$, which always exceeds unity but approaches it in the limit $\alpha \rightarrow \infty$. Thus, the $K$ distribution is not valid under weak irradiance fluctuations for which the scintillation index is less than unity. One attempt at extending the $K$ distribution to the case of weak fluctuations led to the $I-K$ distribution. ${ }^{12,13}$ However, shortcomings in both the $K$ and $I-K$ models have been noted when comparing these pdf's with measured irradiance data in extended turbulence. ${ }^{8}$

Later models arising out of an assumed modulation process were the log-normally modulated exponential distribution $^{14}$ (valid only under strong fluctuation conditions) and the log-normal Rician distribution, also called $^{4,5,8,15}$ Beckmann's pdf. Both of these models show excellent agreement with experimental and simulation data concerning ${ }^{4,5,8}$ the pdf. The more general of the two models is the Beckmann pdf defined by the integral

$$
\begin{aligned}
p(I)= & \frac{(1+r) \exp (-r)}{\sqrt{2 \pi} \sigma_{z}} \times \int_{0}^{\infty} I_{0}\left\{2\left[\frac{(1+r) r I}{z}\right]^{1 / 2}\right\} \\
& \times \exp \left\{-\frac{(1+r) I}{z}-\frac{\left[\ln z+(1 / 2) \sigma_{z}^{2}\right]^{2}}{2 \sigma_{z}^{2}}\right\} \frac{\mathrm{d} z}{z^{2}}, \\
& I>0,
\end{aligned}
$$

where $z$ is mean irradiance, $r$ is a power ratio, $\sigma_{z}^{2}$ is the variance of the lognormal modulation factor $\ln z$, and $I_{0}(x)$ is a modified Bessel function. Although it provides an excellent fit to various data, the Beckmann pdf has certain impediments from a practical point of view. For instance, a closed-form solution for this integral is unknown. Moreover, the poor convergence properties of its integral form makes the Beckmann pdf cumbersome for numerical calculations used in calculating detection or fade probabilities that are essential in understanding the performance characteristics of an optical communications or radar system. Perhaps the most serious impediment to its use is that it is unknown at this time how to deduce the parameters $r$ and $\sigma_{z}^{2}$ of the Beckmann pdf directly from atmospheric parameters.

\section{Gamma-Gamma Distribution}

The pdf model we propose in this paper is based on a modulation process similar to that of the $K$ distribution and the Beckmann pdf. To calculate the parameters of this pdf using only measured values of $C_{n}^{2}$ and $l_{0}$, we use a recently published heuristic theory of scintillation that is applicable for optical wave propagation through all conditions of irradiance fluctuations. ${ }^{16-18}$ Because it is central to the development of our pdf model, we begin this section with a brief review of this heuristic theory.

An optical wave propagating through atmospheric turbulence will experience irradiance fluctuations (scintillation) due to small index of refraction fluctuations commonly 
called optical turbulence. Theoretical and experimental studies of irradiance fluctuations generally involve the scintillation index

$\sigma_{I}^{2}=\frac{\left\langle I^{2}\right\rangle}{\langle I\rangle^{2}}-1$

where the quantity $I$ denotes irradiance (intensity) of the optical wave and the angle brackets \langle\rangle denote an ensemble average or, equivalently with the assumption of ergodicity, a long-time average. Weak fluctuation regimes lead to expressions for the scintillation index that are proportional to the Rytov variance

$\sigma_{1}^{2}=1.23 C_{n}^{2} k^{7 / 6} L^{11 / 6}$

where $k=2 \pi / \lambda$ is the optical wave number, $\lambda$ is wavelength, and $L$ is the propagation path length between transmitter and receiver. For values less than unity, the Rytov variance is the scintillation index of a plane wave in the absence of inner scale effects, and for values greater than unity, it is considered a measure of the strength of optical fluctuations.

Under weak fluctuation conditions, the scintillation index [Eq. (6)] increases with increasing values of the Rytov variance [Eq. (7)]. The scintillation index continues to increase beyond the weak fluctuation regime and reaches a maximum value greater than unity (sometimes as large as 5 or 6) in the regime characterized by random focusing. ${ }^{19}$ With increasing path length or inhomogeneity strength, the focusing effect is weakened by multiple self-interference and the fluctuations slowly begin to decrease, saturating at a level for which the scintillation index approaches unity from above.

In a recent series of papers on scintillation theory, ${ }^{16-18}$ the irradiance of the received optical wave is modeled as a product $I=x y$, where $x$ arises from large-scale turbulent eddies and $y$ from small scale eddies. It is assumed that $x$ and $y$ are statistically independent random processes for which the second moment of irradiance is

$\left\langle I^{2}\right\rangle=\left\langle x^{2}\right\rangle\left\langle y^{2}\right\rangle=\left(1+\sigma_{x}^{2}\right)\left(1+\sigma_{y}^{2}\right)$

where $\sigma_{x}^{2}$ and $\sigma_{y}^{2}$ are normalized variances of $x$ and $y$, respectively. For mathematical convenience, we have taken $\langle I\rangle=1$. Based on Eqs. (6) and (8), the implied scintillation index is

$\sigma_{I}^{2}=\left(1+\sigma_{x}^{2}\right)\left(1+\sigma_{y}^{2}\right)-1=\sigma_{x}^{2}+\sigma_{y}^{2}+\sigma_{x}^{2} \sigma_{y}^{2}$.

Small-scale contributions to scintillation are associated with turbulent cells smaller than the Fresnel zone $R_{F}$ $=(L / k)^{1 / 2}$ or the coherence radius $\rho_{0}$, whichever is smaller. Large-scale fluctuations in the irradiance are generated by turbulent cells larger than that of the first Fresnel zone or the scattering disk $L / k \rho_{0}$, whichever is larger. Under strong fluctuation conditions, spatial cells having size between those of the coherence radius and the scattering disk contribute little to scintillation. That is, because of the loss of spatial coherence, only the very largest cells nearer to the transmitter have any focusing effect on the illumina- tion of small diffractive cells nearer to the receiver, and eventually even these large cells cannot focus or defocus. When this loss of coherence happens, the illumination of the small cells is (statistically) evenly distributed and the fluctuations of the propagating wave are just due to random interference of a large number of diffraction scatterings of the small cells.

To develop a pdf model of the irradiance consistent with this theory, we make the assumption that both large-scale and small-scale irradiance fluctuations are governed by gamma distributions, namely

$$
\begin{aligned}
& p_{x}(x)=\frac{\alpha(\alpha x)^{\alpha-1}}{\Gamma(\alpha)} \exp (-\alpha x), \quad x>0, \quad \alpha>0, \\
& p_{y}(y)=\frac{\beta(\beta y)^{\beta-1}}{\Gamma(\beta)} \exp (-\beta y), \quad y>0, \quad \beta>0 .
\end{aligned}
$$

By first fixing $x$ and writing $y=I / x$, we obtain the conditional pdf

$p_{y}(I \mid x)=\frac{\beta(\beta I / x)^{\beta-1}}{x \Gamma(\beta)} \exp (-\beta I / x), \quad I>0$,

in which $x$ is the (conditional) mean value of $I$. To obtain the unconditional irradiance distribution, we form the average of Eq. (12) over the gamma distribution of Eq. (10), which leads to

$$
\begin{aligned}
p(I) & =\int_{0}^{\infty} p_{y}(I \mid x) p_{x}(x) \mathrm{d} x \\
& =\frac{2(\alpha \beta)^{(\alpha+\beta) / 2}}{\Gamma(\alpha) \Gamma(\beta)} I^{(\alpha+\beta) / 2-1} K_{\alpha-\beta}\left[2(\alpha \beta I)^{1 / 2}\right], \quad I>0 .
\end{aligned}
$$

We call Eq. (13) the gamma-gamma distribution. The positive parameter $\alpha$ represents the effective number of large-scale cells of the scattering process and $\beta$ similarly represents the effective number of small-scale cells. When optical turbulence is weak, the effective number of scale sizes smaller and larger than the first Fresnel zone is large, resulting in $\alpha \gg 1$ and $\beta \gg 1$. As the irradiance fluctuations increase and the focusing regime is approached, both parameters of Eq. (13) decrease substantially. Beyond the focusing regime and approaching the saturation regime, we find that $\beta \rightarrow 1$, indicating that the effective number of small-scale cells ultimately reduces to one, determined by the transverse spatial coherence radius of the optical wave. On the other hand, the effective number of discrete refractive scatterers $\alpha$ increases again with increasing turbulence strength and eventually becomes unbounded in the saturation regime. Under these conditions, the gamma-gamma distribution approaches the negative exponential distribution in the deep saturation regime.

From the gamma-gamma pdf [Eq. (13)] we find $\left\langle I^{2}\right\rangle$ $=(1+1 / \alpha)(1+1 / \beta)$, and thus we identify the parameters of this distribution with the large-scale and small-scale scintillation according to 
$\alpha=\frac{1}{\sigma_{x}^{2}}, \quad \beta=\frac{1}{\sigma_{y}^{2}}$.

It follows that the total scintillation index [Eq. (9)] is related to these parameters by

$\sigma_{I}^{2}=\frac{1}{\alpha}+\frac{1}{\beta}+\frac{1}{\alpha \beta}$.

\section{Comparison with Plane Wave Data}

In this section, we compare the gamma-gamma distribution model with published numerical simulation data for the pdf by Flatté et al. ${ }^{3}$ The simulation data for a plane wave incident on a random medium characterized by homogeneous, isotropic Kolmogorov turbulence led to numerous plots of the $\log$-irradiance pdf as a function of $(\ln I-\langle\ln I\rangle) / \sigma$, covering a range of conditions that extends from weak irradiance fluctuations well into the saturation regime characterized by $\sigma_{1}^{2}=25$. Here, $\langle\ln I\rangle$ is the mean value of the $\log$ irradiance and $\sigma=\sqrt{\sigma_{\ln I}^{2}}$, the latter being the root mean square (rms) value of $\ln I$. The simulation pdf's were displayed in this fashion in the hope that it would reveal their salient features. In the saturation regime the simulation data of Flatte et al. ${ }^{3}$ showed that the plane wave pdf lies somewhere between the log-normal and exponential distributions, and their moments lie between those of a log-normalexponential distribution and those of a $K$ distribution.

The model for the refractive-index spectrum used to generate the simulation data was of the form

$\Phi_{n}(\kappa)=0.033 C_{n}^{2} \kappa^{-11 / 3} f\left(\kappa l_{0}\right)$,

where $f\left(\kappa l_{0}\right)$ is a nondimensional function that describes the high wave number spectral bump and dissipation range. ${ }^{20}$ Although the analytic form of this nondimensional function is unknown, Andrews ${ }^{21}$ has shown that it can be closely approximated by

$$
\begin{aligned}
f\left(\kappa l_{0}\right)= & \exp \left(-\kappa^{2} / \kappa_{l}^{2}\right)\left[1+1.802\left(\kappa / \kappa_{l}\right)\right. \\
& \left.-0.254\left(\kappa / \kappa_{l}\right)^{7 / 6}\right], \quad \kappa_{l}=3.3 / l_{0}
\end{aligned}
$$

In past analyses it was common to use the traditional spectrum in which $f\left(\kappa l_{0}\right)=\exp \left[-\left(\kappa l_{0} / 5.92\right)^{2}\right]$, but Flatté et al. ${ }^{3}$ point out that this latter spectrum leads to inaccuracies by as much as $50 \%$ for predicting the scintillation index.

For negligible inner scale, the scintillation theory in Refs. 16 and 18 for a plane wave leads to the large-scale and small-scale variances given, respectively, by

$$
\begin{aligned}
& \sigma_{x}^{2} \cong \exp \left[\frac{0.49 \sigma_{1}^{2}}{\left(1+1.11 \sigma_{1}^{12 / 5}\right)^{7 / 6}}\right]-1, \\
& \sigma_{y}^{2} \cong \exp \left[\frac{0.51 \sigma_{1}^{2}}{\left(1+0.69 \sigma_{1}^{12 / 5}\right)^{7 / 6}}\right]-1 .
\end{aligned}
$$

In the presence of a finite inner scale, the comparable expression for the large-scale scintillation is

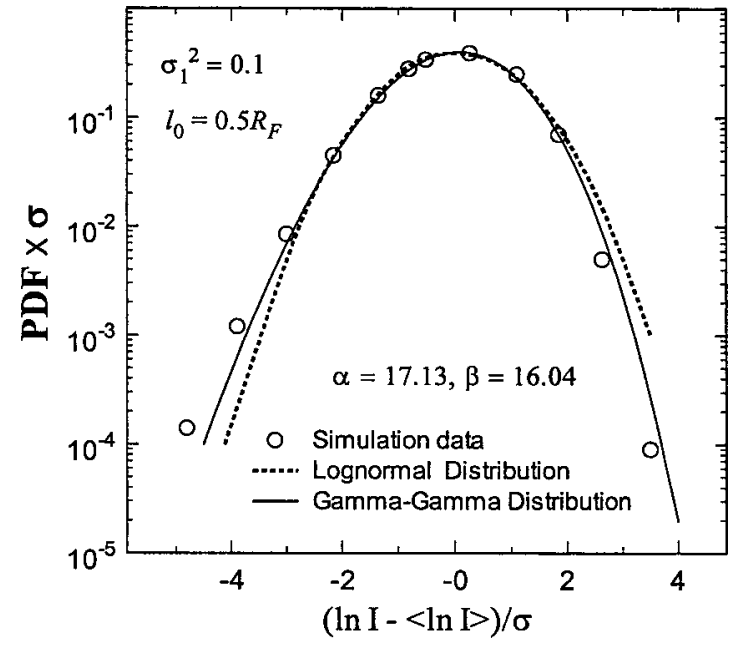

Fig. 1 The pdf of the scaled log-irradiance for a plane wave in the case of weak irradiance fluctuations: $\sigma_{1}^{2}=0.1$ and $I_{0} / R_{F}=0.5$. The open circles represent simulation data, the dashed line is from the lognormal pdf, and the solid line is from the gamma-gamma pdf [Eq. (13)] with $\alpha$ and $\beta$ predicted by Eqs. (14).

$$
\begin{aligned}
\sigma_{x}^{2}= & \exp \left\{0.16 \sigma_{1}^{2}\left(\frac{2.61 \eta_{l}}{2.61+\eta_{l}+0.45 \sigma_{1}^{2} \eta_{l}^{7 / 6}}\right)^{7 / 6}\right. \\
& \times\left[1+1.753\left(\frac{2.61}{2.61+\eta_{l}+0.45 \sigma_{1}^{2} \eta_{l}^{7 / 6}}\right)^{1 / 2}\right. \\
& \left.\left.-0.252\left(\frac{2.61}{2.61+\eta_{l}+0.45 \sigma_{1}^{2} \eta_{l}^{7 / 6}}\right)^{7 / 12}\right]\right\}-1,
\end{aligned}
$$

where $\eta_{l}=L \kappa_{l}^{2} / k l_{0}^{2}=10.89\left(R_{F} / l_{0}\right)^{2}$, and the small-scale variance is still approximated by Eq. (19). From these expressions the parameters $\alpha$ and $\beta$ of the gamma-gamma pdf can be determined through the use of Eq. (14). The selection of parameters based on Eq. (14) is equivalent to using only measured values of the refractive index structure parameter $C_{n}^{2}$ and inner scale $l_{0}$ to predict all other parameters arising in scaling the plots of the simulation data.

We plot in Figs. 1-5 the predicted log-irradiance pdf associated with the gamma-gamma distribution (solid line) for comparison with the simulation data illustrated in Figs. 4, 5, and 7 of Ref. 3. As representative of typical atmospheric propagation conditions, we use the inner scale value $l_{0}=0.5 R_{F}\left(\eta_{l}=44\right)$ in Figs. 1 and 2 . In Fig. 1, we use the simulation values $\sigma_{1}^{2}=0.1, l_{0}=0.5 R_{F}$, and in Fig. 2 we use the simulation values $\sigma_{1}^{2}=2, l_{0}=0.5 R_{F}$, taken from Figs. 4 and 5, respectively, of Ref. 3. We also plot the log-normal pdf (dashed line) in Fig. 1 for the sake of comparison. Here we find that the gamma-gamma distribution provides a better fit to the simulation data than does the log-normal distribution. In fact, the predicted gammagamma pdf generally provides a good fit to the data over most of the abscissa values in Figs. 1 and 2, except it falls somewhat under the data for values on the abscissa less than -3 . Rather than use the simulation results, however, values of the scaling parameters $\langle\ln I\rangle$ and $\sigma$ required in the plots were calculated directly from the gamma-gamma pdf. 


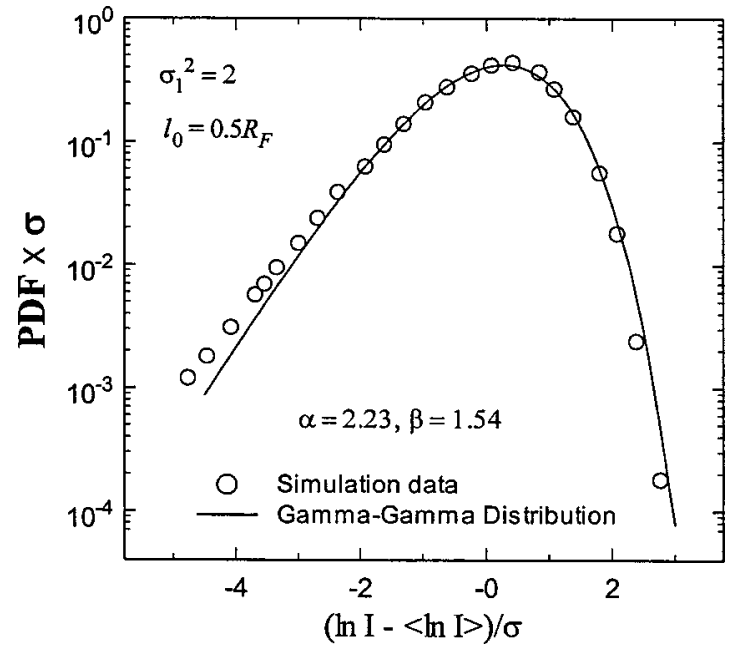

Fig. 2 Same as Fig. 1 with $\sigma_{1}^{2}=2$, corresponding to moderate irradiance fluctuations.

To further test the gamma-gamma model under a variety of inner scale conditions, we considered the three cases presented in Fig. 7 of Ref. 3. In Fig. 3 we use the simulation values $\sigma_{1}^{2}=25, l_{0}=0$, to predict the parameters $\alpha$ and $\beta$ of the gamma-gamma pdf. This value of the Rytov variance corresponds to the saturation regime, in which our model predicts a scintillation index of $\sigma_{I, \mathrm{pl}}^{2}=1.214$ as compared with the higher simulation value $\sigma_{I, \mathrm{pl}}^{2}=1.387$. We also plot the $K$ distribution using the simulation data for identifying its free parameter. In this case both distributions provide an excellent fit to the simulation data. In Figs. 4 and 5 we use the simulation parameter values $\sigma_{1}^{2}=25, l_{0}$ $=0.5 R_{F}$, and $\sigma_{1}^{2}=25, l_{0}=R_{F}\left(\eta_{l}=11\right)$, which correspond to the saturation regime but now with inner scale effects. The predicted scintillation index is found to be $\sigma_{I, \mathrm{pl}}^{2}$

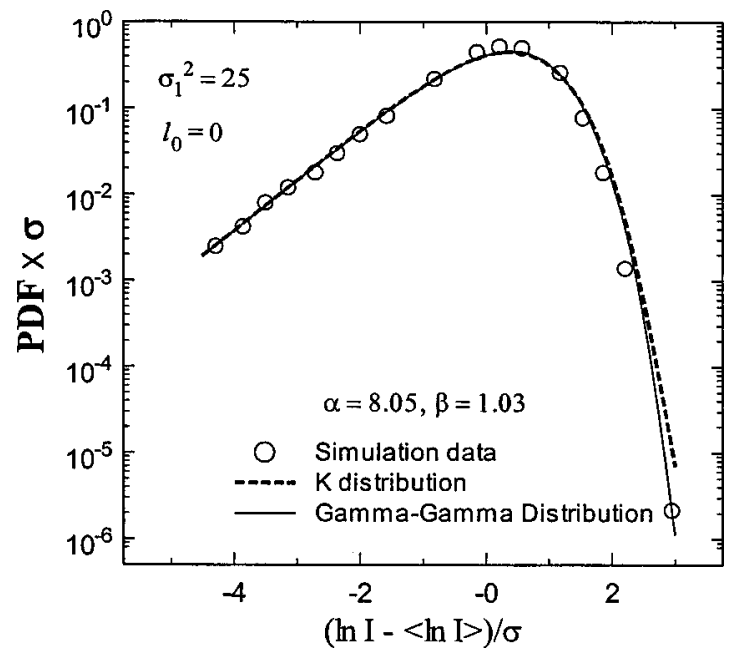

Fig. 3 The pdf of the scaled log-irradiance for a plane wave in the case of strong irradiance fluctuations: $\sigma_{1}^{2}=25$ and $I_{0} / R_{F}=0$. The open circles represent simulation data, the dashed line is the $K$ distribution [Eq. (4)] with parameter $\alpha$ determined from the simulation data, and the solid line is from the gamma-gamma pdf [Eq. (13)] with $\alpha$ and $\beta$ predicted by Eqs. (14).

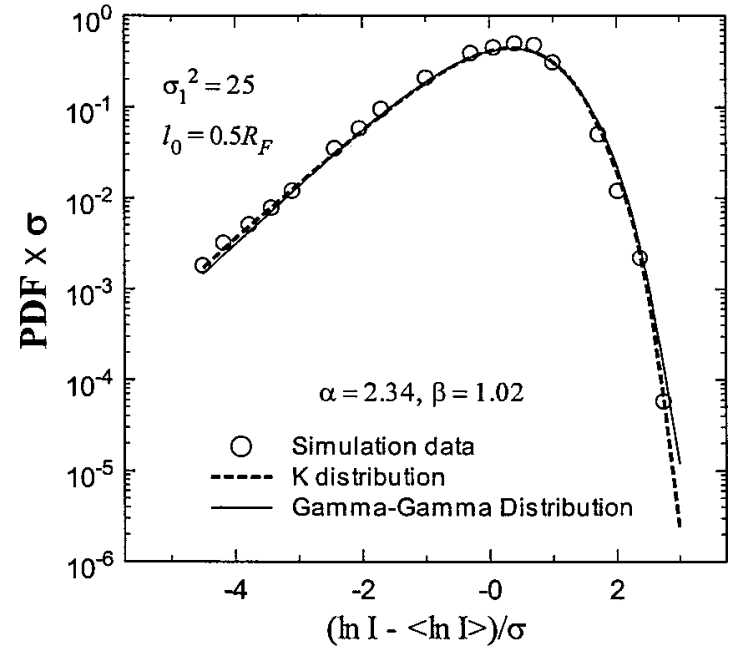

Fig. 4 Same as Fig. 3 with $I_{0} / R_{F}=0.5$.

$=1.823$ and $\sigma_{I, \mathrm{pl}}^{2}=2.246$, respectively, whereas the simulation values are somewhat lower, namely, $\sigma_{I, \mathrm{pl}}^{2}=1.554$ and $\sigma_{I, \mathrm{pl}}^{2}=1.841$. However, once again both the gamma-gamma and $K$ distributions show excellent fits with the pdf simulation data. We do note that in Figs. 3 to 5 the gammagamma parameter $\beta \cong 1$, which means there is little difference between the gamma-gamma pdf and the $K$ distribution in this regime.

\section{Comparison with Spherical Wave Data}

Hill et al. ${ }^{4}$ and Hill and Frehlich ${ }^{5}$ used numerical simulation of the propagation of a spherical wave through homogeneous and isotropic atmospheric turbulence that also led to pdf data for the log-irradiance fluctuations. In that analysis, they compared several heuristic pdf models with the numerical data and concluded that the Beckmann distribution provided the closest fit over all conditions tested. On the other hand, their simulation data did not generally support the $K$ distribution.

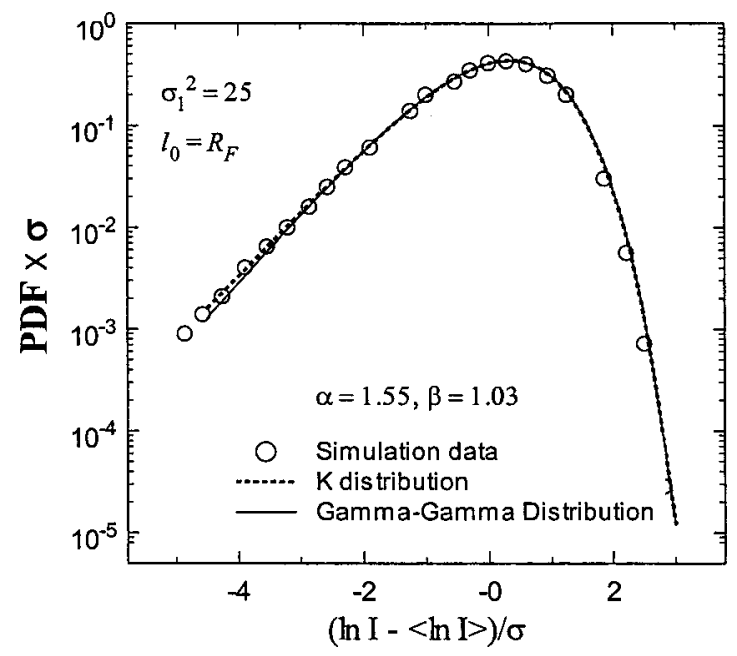

Fig. 5 Same as Fig. 3 with $I_{0} / R_{F}=1$. 
Various additional parameters used in the analysis of the numerical simulation data for a spherical wave include the following:

$\beta_{0}^{2}=0.4 \sigma_{1}^{2}=0.496 C_{n}^{2} k^{7 / 6} L^{11 / 6}$,

$\widetilde{\sigma}^{2}\left(l_{0} / R_{F}\right)=10.5 \int_{0}^{1} \mathrm{~d} u \int_{0}^{\infty} \mathrm{d} x x^{-8 / 3} f\left(x l_{0} / R_{F}\right)$

$\times \sin ^{2}\left[x^{2} u(1-u) / 2\right]$,

$\sigma_{\text {Rytov }}^{2}=\beta_{0}^{2} \widetilde{\sigma}^{2}\left(l_{0} / R_{F}\right)$.

The quantity $\beta_{0}^{2}$ is the classic Rytov scintillation index of a spherical wave in the limit of weak scintillation and a Kolmogorov spectrum, and the Rytov parameter [Eq. (23)] is the weak fluctuation scintillation index in the presence of a finite inner scale. The parameter $\sigma^{2}\left(l_{0} / R_{F}\right)$ defined by [Eq. (22)] is the ratio between these two Rytov parameters. In our calculation of $\beta_{0}^{2}$ we used the expression ${ }^{22,23}$

$$
\begin{aligned}
\widetilde{\sigma}^{2}\left(l_{0} / R_{F}\right) \cong & 3.86\left\{( 1 + 9 / \eta _ { l } ^ { 2 } ) ^ { 1 1 / 1 2 } \left[\sin \left(\frac{11}{6} \tan ^{-1} \frac{\eta_{l}}{3}\right)\right.\right. \\
& +\frac{2.610}{\left(9+\eta_{l}^{2}\right)^{1 / 4}} \sin \left(\frac{4}{3} \tan ^{-1} \frac{\eta_{l}}{3}\right) \\
& \left.\left.-\frac{0.518}{\left(9+\eta_{l}^{2}\right)^{7 / 24}} \sin \left(\frac{5}{4} \tan ^{-1} \frac{\eta_{l}}{3}\right)\right]-8.75 \eta_{l}^{-5 / 6}\right\} .
\end{aligned}
$$

Based on the scintillation theory given in Refs. 16-18, the large-scale and small-scale scintillations in the absence of inner scale are described by

$\sigma_{x}^{2} \cong \exp \left[\frac{0.49 \beta_{0}^{2}}{\left(1+0.56 \beta_{0}^{12 / 5}\right)^{7 / 6}}\right]-1$,

$\sigma_{y}^{2} \cong \exp \left[\frac{0.51 \beta_{0}^{2}}{\left(1+0.69 \beta_{0}^{12 / 5}\right)^{7 / 6}}\right]-1$

which bear a strong resemblance to Eqs. (18) and (19) for the plane wave, but this time with the Rytov variance $\beta_{0}^{2}$. When inner scale effects are considered, the small-scale scintillation is again described by Eq. (26) and the comparable large-scale expression is

$$
\begin{aligned}
\sigma_{x}^{2} \cong & \exp \left\{0.04 \beta_{0}^{2}\left(\frac{8.56 \eta_{l}}{8.56+\eta_{l}+0.195 \beta_{0}^{2} \eta_{l}^{7 / 6}}\right)^{7 / 6}\right. \\
& \times\left[1+1.753\left(\frac{8.56}{8.56+\eta_{l}+0.195 \beta_{0}^{2} \eta_{l}^{7 / 6}}\right)^{1 / 2}\right. \\
& \left.\left.-0.252\left(\frac{8.56}{8.56+\eta_{l}+0.195 \beta_{0}^{2} \eta_{l}^{7 / 6}}\right)^{7 / 12}\right]\right\}-1 .
\end{aligned}
$$

Parameters of the gamma-gamma pdf are readily deduced from these expressions using Eq. (14) and the total scintillation index is once again obtained from Eq. (9).

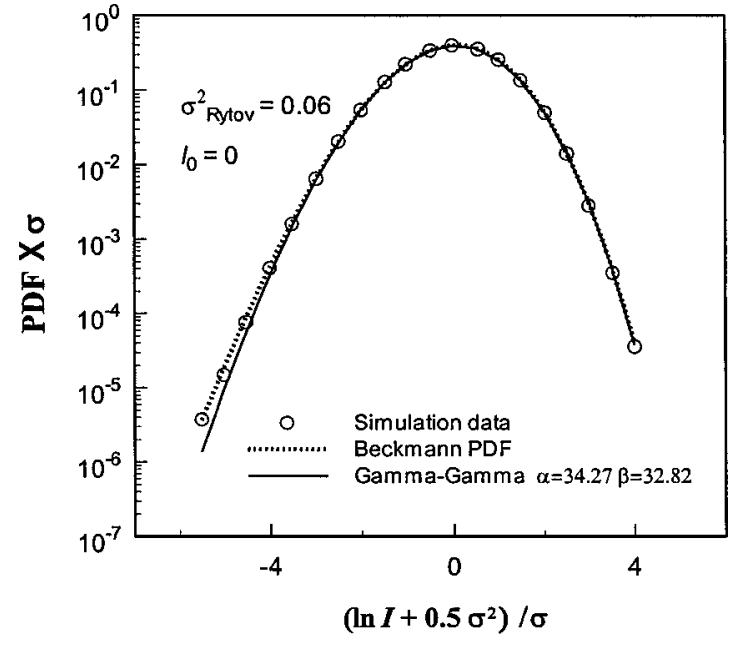

Fig. 6 The pdf of the scaled log-irradiance for a spherical wave in the case of weak irradiance fluctuations: $\sigma_{\text {Rytov }}^{2}=0.06$ and $I_{0} / R_{F}$ $=0$. The open circles represent simulation data, the dotted line is from Beckmann's pdf [Eq. (5)], and the solid line is from the gammagamma pdf [Eq. (13)] with $\alpha$ and $\beta$ predicted by Eqs. (14).

Following Refs. 4 and 12, we plot in Figs. 6-14 simulation pdf data and pdf values predicted by the Beckmann distribution (dotted line) as a function of $\left(\ln I+0.5 \sigma^{2}\right) / \sigma$ and $\sigma_{\text {Rytov }}^{2}=0.06,2$, and 5. Values of $r$ and $\sigma_{z}^{2}$ required by the Beckmann pdf [Eq. (5)] were provided in tabular form in Ref. 4 for various values of $l_{0} / R_{F}$ and Rytov parameter $\sigma_{\text {Rytov }}^{2}$. Also shown in Figs. 6-14 are curves deduced from the gamma-gamma distribution (solid line).

In Figs. 6-8, the inner scale is negligibly small. The gamma-gamma pdf curves in Figs. 6 and 8 provide very good fits with the data, but fairly large deviations in the scaled pdf can be seen in Fig. 7 for values along the left side of the abscissa (i.e., small irradiance values). We found that by incrementing the parameters $\alpha$ and $\beta$ of the gammagamma pdf we could produce a "best fit" curve (dashed line) that is comparable to the fit of Beckmann's pdf. This

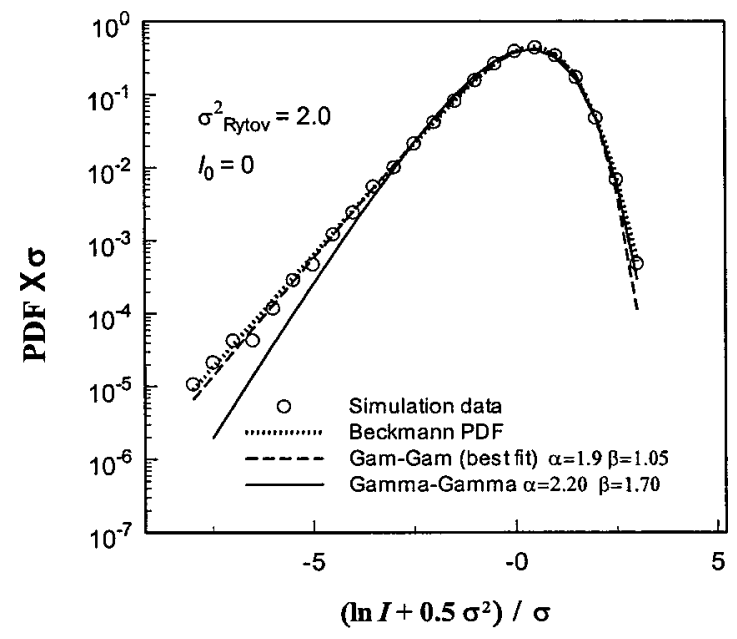

Fig. 7 Same as Fig. 6 with $\sigma_{\text {Rytov }}^{2}=2$ corresponding to moderate irradiance fluctuations. Also shown (dashed line) is the gammagamma pdf with parameters $\alpha$ and $\beta$ chosen from a best fit to the data. 


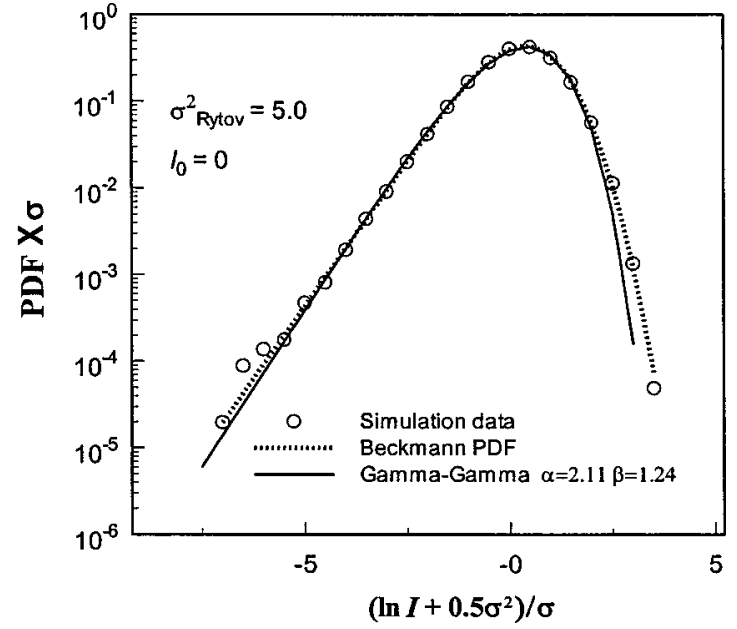

Fig. 8 Same as Fig. 6 with $\sigma_{\text {Rytov }}^{2}=5$ corresponding to strong irradiance fluctuations.

suggests that the gamma-gamma pdf is applicable here, but the scintillation theory ${ }^{16-18}$ used to predict $\alpha$ and $\beta$ apparently underestimates both large-scale and small-scale scintillations in this case.

The curves and data in Figs. 9-14 are similar to that in Figs. 6-8 except that $l_{0} / R_{F}=0.5\left(\eta_{l}=44\right)$ in Figs. 9-11 and $l_{0} / R_{F}=1\left(\eta_{l}=11\right)$ in Figs. $12-14$. For the case of $\sigma_{\text {Rytov }}^{2}=5$ in Figs. 11 and 14, the Beckmann pdf did not lend itself directly to numerical calculations and so is omitted. ${ }^{4}$ With the exception of Fig. 14, we believe the gamma-gamma pdf once again shows good agreement with the data. In the exceptional case we incremented the parameters of the gamma-gamma pdf as in Fig. 7 to obtain the best fit curve shown in Fig. 14 (dashed line).

\section{Discussion}

Flatté et al. ${ }^{3}$ calculated the pdf from simulations for a plane wave propagated through atmospheric turbulence and compared the results with several pdf models. In the case of the plane wave data, it was concluded that the simulation data lie closest to the $K$ distribution in strong turbulence. The $K$

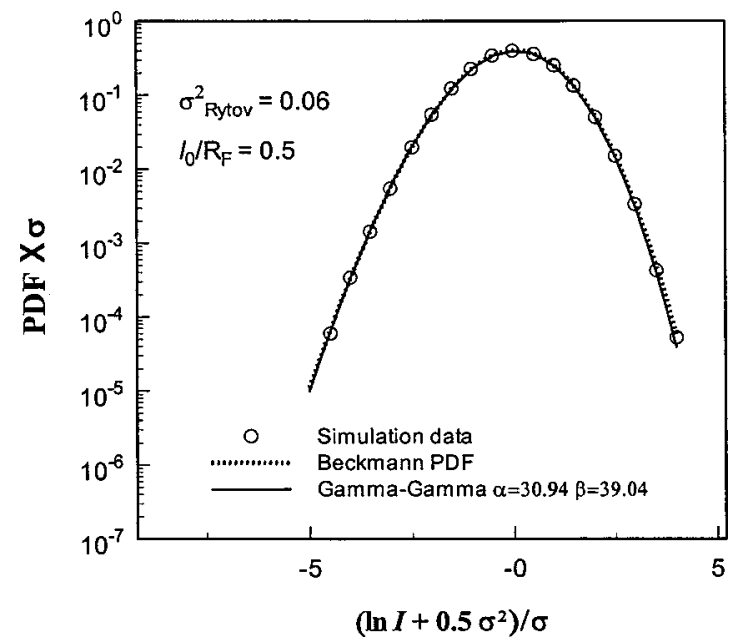

Fig. 9 Same as Fig. 6 with $I_{0} / R_{F}=0.5$.

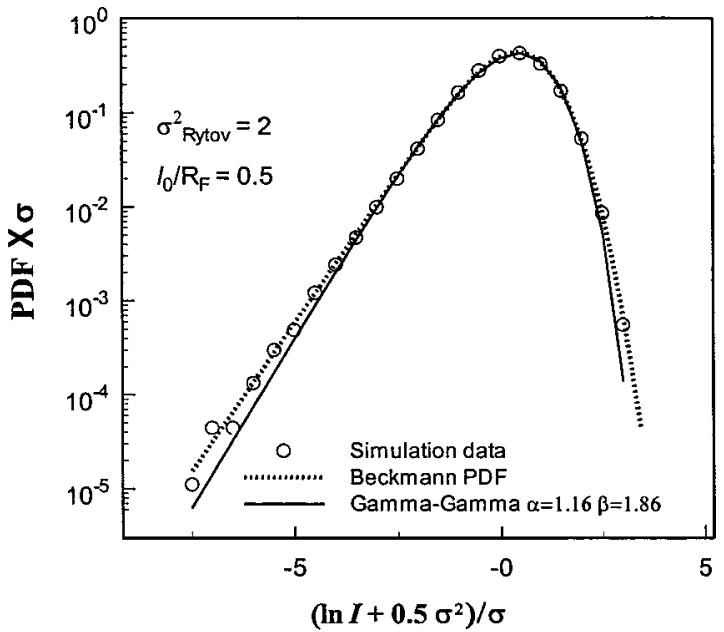

Fig. 10 Same as Fig. 7 with $I_{0} / R_{F}=0.5$.

distribution has one free parameter that depends on the value of some moment from the simulation data like $\langle\ln I\rangle$ or $\sigma_{I}^{2}$. The lognormal model, which was a universal model in the scaled plots, was shown in all figures of Ref. 3 for comparison. However, even in weak fluctuations the lognormal model did not fit the data well in the tails of the distribution.

In our analysis of the data in Ref. 3 the gamma-gamma PDF fit the simulation data better than the log-normal model fit the data in weak fluctuations (Fig. 1), but the gamma-gamma model still lies somewhat off the data in the extreme tails in both Figs. 1 and 2. Under strong fluctuations (Figs. 3-5), there is little distinction between the $K$ distribution and gamma-gamma distribution, and both provide an excellent fit with all the data even for changes in inner-scale values.

Hill et al. ${ }^{4}$ and Hill and Frehlich ${ }^{5}$ did a numerical simulation analysis for an initially spherical wave similar to that by Flatte et al. ${ }^{3}$ and found the best agreement with the simulation data was provided by Beckmann's pdf [Eq. (5)].

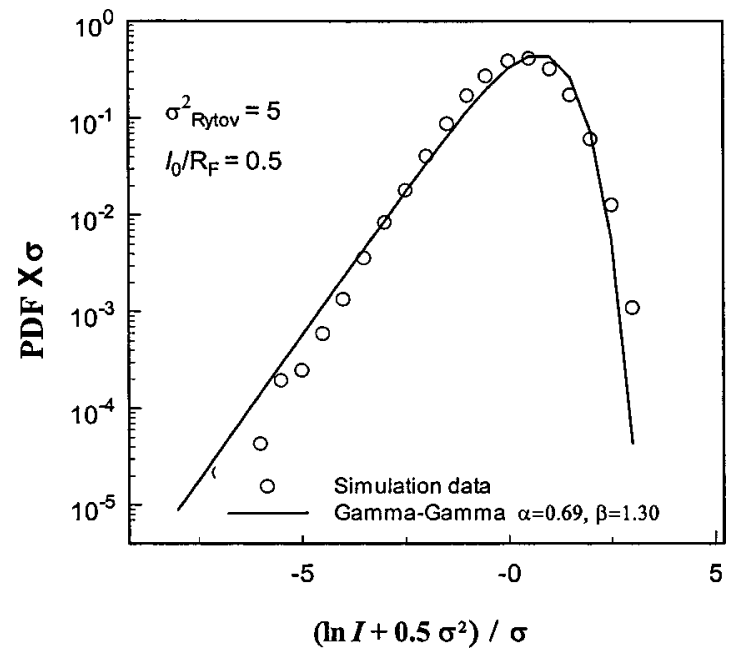

Fig. 11 Same as Fig. 8 with $I_{0} / R_{F}=0.5$ but without the Beckmann pdf. 


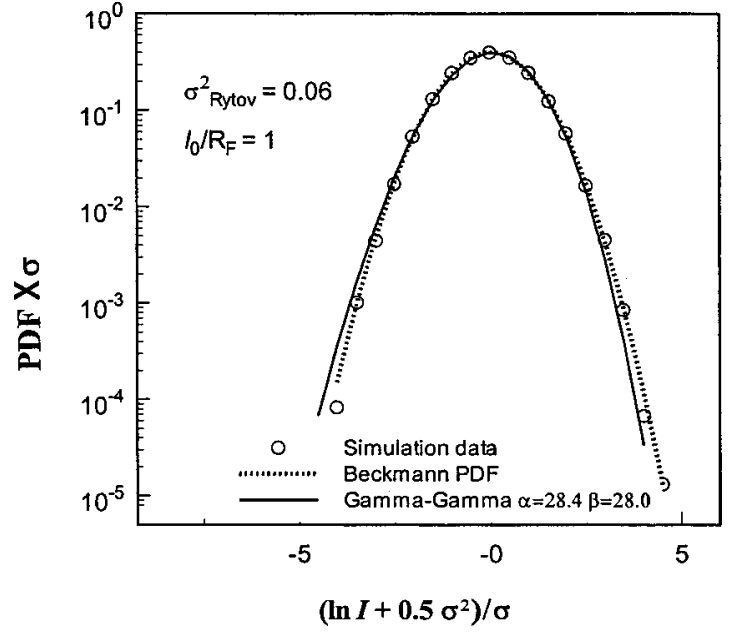

Fig. 12 Same as Fig. 6 with $I_{0} / R_{F}=1$.

Like other models, the parameters $r$ and $\sigma_{z}^{2}$ of Beckmann's pdf were not directly related to conditions of optical turbulence, such as $C_{n}^{2}$ and inner scale $l_{0}$, but were determined instead by the simulations' values of $\langle\ln I\rangle$ and $\left\langle I^{2}\right\rangle$ except for the case illustrated in our Fig. 8 in which they used $\left\langle I^{-1 / 2}\right\rangle$ and $\left\langle I^{2}\right\rangle$. Doing so enabled these researchers to fit the Beckmann pdf with one statistic that is determined by relatively smaller values of irradiance, namely, $\langle\ln I\rangle$ or $\left\langle I^{-1 / 2}\right\rangle$, and one statistic that is determined by larger values of irradiance, namely, $\left\langle I^{2}\right\rangle$. Beckmann's pdf does not permit determination of its parameters from the simulation statistics involving any combination of $\langle\ln I\rangle,\left\langle I^{-1 / 2}\right\rangle$, and $\left\langle I^{2}\right\rangle$ for the cases shown in our Figs. 11 and 14 involving nonzero inner scale values and $\sigma_{\text {Rytov }}^{2}=5$. Nonetheless, a fit could be forced by some other means as pointed out by Hill and Frehlich. ${ }^{5}$

Except for Figs. 7 and 14, our analysis showed that the gamma-gamma pdf provides a good fit to the spherical wave simulation data for the given inner scale values and calculated values of $\beta_{0}^{2}$, but generally did not fit as well as the Beckmann model. Nonetheless, we believe that detec-

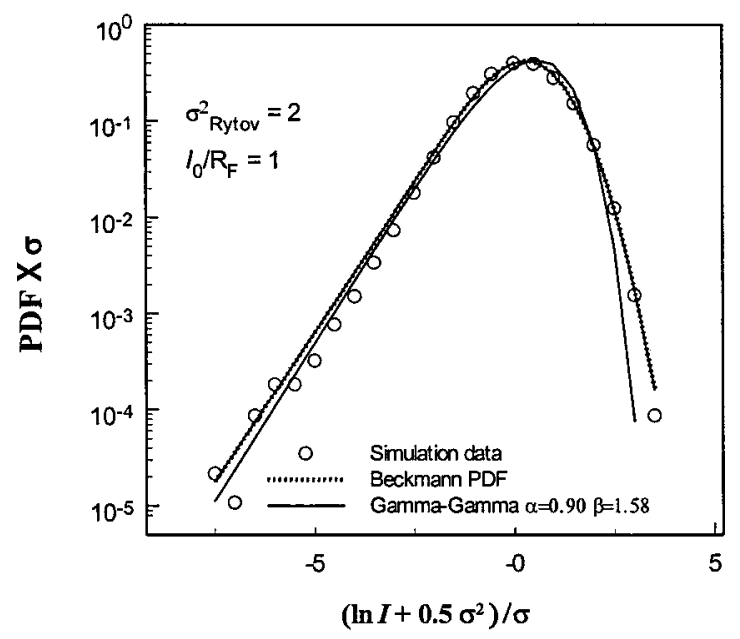

Fig. 13 Same as Fig. 7 with $I_{0} / R_{F}=1$.

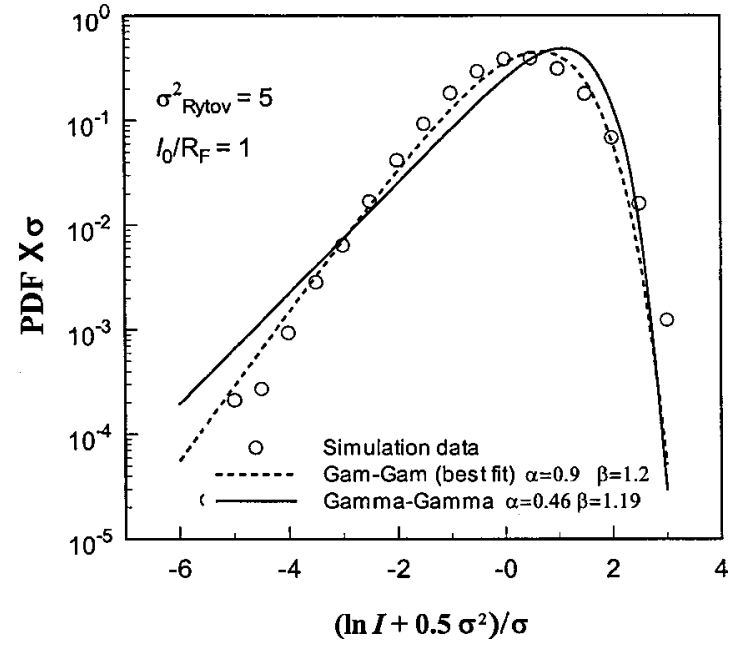

Fig. 14 Same as Fig. 8 with $I_{0} / R_{F}=1$ but without the Beckmann pdf. Also shown (dashed line) is the gamma-gamma pdf with parameters $\alpha$ and $\beta$ chosen from a best fit to the data.

tion or fade probabilities deduced from the gamma-gamma model will provide useful information about the performance characteristics of various lasercom systems. Also, an advantage to using the gamma-gamma pdf model is that the parameters can be estimated directly from atmospheric data.

It has recently been pointed out by Flatté and Gerber ${ }^{24}$ that the asymptotic theory ${ }^{25}$ on which the heuristic scintillation theory ${ }^{16-18}$ is based does not have quite the same power law behavior near the saturation regime as that of the simulation data. This difference in theoretical and simulation data power laws under moderate-to-strong irradiance fluctuations may account for the differences we observed in some of the comparisons made here, particularly in Figs. 7 and 14 . We are currently investigating a modification in the scintillation model ${ }^{16-18}$ by considering outer scale effects. Our preliminary findings suggest that a finite outer scale may indeed account for the difference between simulation data and current theory in moderate-to-strong fluctuations, i.e., outer scale effects are inherent in simulation data based on numerical grid size. Thus, although the current scintillation theory ${ }^{16-18}$ may not always predict optimum values of parameters $\alpha$ and $\beta$, this should not be interpreted as a shortcoming of the gamma-gamma pdf model. In fact, we believe the results presented in Figs. 1-14 already validate this pdf as a practical model for irradiance fluctuations.

Last, let us point out that the gamma-gamma distribution derived here is not a new pdf. Along with the gamma distribution (sometimes called the $m$-distribution), the gammagamma pdf appears in the work of Nakagami ${ }^{26}$ and was proposed as an irradiance model for polychromatic and partially developed speckle after propagating through atmospheric turbulence. ${ }^{27}$ We have suggested the gammagamma pdf in the present work as a reasonable alternative to Beckmann's pdf for a number of reasons. First, the gamma distribution itself has often been proposed as an approximation to the lognormal model and the Rician ${ }^{26-28}$ pdf, the latter models used to define Beckmann's pdf. It is desirable to use the gamma distribution as an approximation to both the lognormal and Rician pdf's because of its 
simple functional form, which leads to a closed-form representation of the gamma-gamma pdf given by Eq. (13). This makes computations extremely easy in comparison with Beckmann's pdf. Second, parameter values for $\alpha$ and $\beta$ of the gamma-gamma pdf can be directly related to calculated values of large-scale and small-scale scintillation that depend only on values of $C_{n}^{2}$ and $l_{0}$; moreover, parameters of the gamma-gamma pdf can always be found by making small adjustments that permit the distribution to fit the simulation data. Third, and perhaps most important, the cumulative distribution function (cdf) for the gammagamma pdf can also be found in closed form. For practical purposes, it is the cdf that is of greater interest than the pdf since the former is used to predict probabilities of detection and fade in an optical communication or radar system. The cdf associated with the gamma-gamma distribution is readily found to be

$$
\begin{aligned}
P\left(I \leqslant I_{T}\right)= & \int_{0}^{I_{T}} p(I) \mathrm{d} I \\
= & \frac{\pi}{\sin [\pi(\alpha-\beta)] \Gamma(\alpha) \Gamma(\beta)}\left\{\frac{\left(\alpha \beta I_{T}\right)^{\beta}}{\beta \Gamma(\beta-\alpha+1)}{ }_{1} F_{2}\right. \\
& \times\left(\beta ; \beta+1, \beta+1, \beta-\alpha+1 ; \alpha \beta I_{T}\right) \\
& -\frac{\left(\alpha \beta I_{T}\right)^{\alpha}}{\alpha \Gamma(\alpha-\beta+1)}{ }_{1} F_{2}(\alpha ; \alpha+1, \alpha+1, \alpha-\beta \\
& \left.\left.+1 ; \alpha \beta I_{T}\right)\right\},
\end{aligned}
$$

where $I_{T}$ is a threshold parameter and ${ }_{1} F_{2}$ denotes a generalized hypergeometric function. ${ }^{29}$

\section{Acknowledgments}

Funding for this work was partially provided by the Ballistic Missile Defense Organization's (BMDO) Innovative Science and Technology Directorate and administrated by the Space and Naval Warfare Systems Center, San Diego, California, under Contract No. N66001-97-C-6008. Also, the authors are deeply indebted to W. D. Otto of the Environmental Technology Laboratory of the National Oceanic and Atmospheric Administration (NOAA) for supplying us with the spherical wave simulation data and data for Beckmann's pdf.

\section{References}

1. S. G. Lambert and W. L. Casey, Laser Communications in Space, Artech House, Boston (1995).

2. B. L. Edelson and G. Hyde, "A report of the IEEE-USA Aerospace Policy Committee on laser satellite communications, programs, technology and applications," IEEE (Apr. 1996).

3. S. M. Flatté, C. Bracher, and G.-Yu Wang, "Probability-density functions of irradiance for waves in atmospheric turbulence calculated by numerical simulations," J. Opt. Soc. Am. A 11, 2080-2092 (1994).

4. R. J. Hill, R. G. Frehlich, and W. D. Otto, "The probability distribution of irradiance scintillation," NOAA Technical Memo ERL ETL274, NOAA Environmental Research Laboratories, Boulder, CO (1996).
5. R. J. Hill and R. G. Frehlich, "Probability distribution of irradiance for the onset of strong scintillation," J. Opt. Soc. Am. A 14, 15301540 (1997).

6. G. Parry, "Measurements of atmospheric turbulence-induced intensity fluctuations in a laser beam," Opt. Acta 28, 715-728 (1981).

7. R. L. Phillips and L. C. Andrews, "Measured statistics of laser-light scattering in atmospheric turbulence," J. Opt. Soc. Am. 71, 14401445 (1981).

8. J. H. Churnside and R. G. Frehlich, "Experimental evaluation of lognormally modulated Rician and $I K$ models of optical scintillation in the atmosphere," J. Opt. Soc. Am. A 6, 1760-1766 (1989).

9. E. Jakeman and P. N. Pusey, "A model for non-Rayleigh sea echo," IEEE Trans. Antennas Propag. AP-24, 806-814 (1976).

10. E. Jakeman and P. N. Pusey, "The significance of $K$-distributions in scattering experiments," Phys. Rev. Lett. 40, 546-550 (1978).

11. E. Jakeman, "On the statistics of $K$-distributed noise," J. Phys. A 13, 31-48 (1980).

12. L. C. Andrews and R. L. Phillips, " $I-K$ distribution as a universal propagation model of laser beams in atmospheric turbulence," $J$. Opt. Soc. Am. A 2, 160-163 (1985).

13. L. C. Andrews and R. L. Phillips, "Mathematical genesis of the $I-K$ distribution for random optical fields," J. Opt. Soc. Am. A 3, 19121919 (1986).

14. J. H. Churnside and R. J. Hill, "Probability density of irradiance scintillations for strong path-integrated refractive turbulence," J. Opt. Soc. Am. A 4, 727-733 (1987).

15. J. H. Churnside and S. F. Clifford, "Log-normal Rician probabilitydensity function of optical scintillations in the turbulent atmosphere," J. Opt. Soc. Am. A 4, 1923-1930 (1987).

16. L. C. Andrews, R. L. Phillips, C. Y. Hopen, and M. A. Al-Habash, "Theory of optical scintillation," J. Opt. Soc. Am. A 16, 1417-1429 (1999).

17. L. C. Andrews, R. L. Phillips, and C. Y. Hopen, "Aperture averaging of optical scintillations: power fluctuations and the temporal spectrum," Waves Random Media 10, 53-70 (2000).

18. L. C. Andrews, R. L. Phillips, and C. Y. Hopen, "Scintillation model for a satellite communication link at large zenith angles," Opt. Eng. 39, 3272-3280 (2000)

19. A. Consortini, R. Cochetti, J. H. Churnside, and R. J. Hill, "Innerscale effect on irradiance variance measured for weak-to-strong atmospheric scintillation," J. Opt. Soc. Am. A 10, 2354-2362 (1993).

20. R. J. Hill and S. F. Clifford, "Modified spectrum of atmospheric temperature fluctuations and its application to optical propagation," $J$. Opt. Soc. Am. 68, 892-899 (1978).

21. L. C. Andrews, "An analytical model for the refractive index power spectrum and its application to optical scintillations in the atmosphere," J. Mod. Opt. 39, 1849-1853 (1992).

22. W. B. Miller, J. C. Ricklin, and L. C. Andrews, "Effects of the refractive index spectral model on the irradiance variance of a Gaussian beam," J. Opt. Soc. Am. A 11, 2719-2726 (1994).

23. L. C. Andrews and R. L. Phillips, Laser Beam Propagation through Random Media, SPIE Optical Engineering Press, Bellingham, WA (1998).

24. S. M. Flatté and J. S. Gerber, "Irradiance-variance behavior by numerical simulation for plane-wave and spherical-wave optical propagation through strong turbulence," J. Opt. Soc. Am. A 17, 1092-1097 (2000).

25. K. S. Gochelashvili and V. I. Shishov, "Saturated fluctuations in the laser radiation intensity in a turbulent medium," Sov. Phys. JETP 39 605-609 (1974)

26. M. Nakagami, "The $m$ distribution-a general formula of intensity distribution of rapid fading." in Statistical Methods in Radio Wave Propagation, W. C. Hoffman, Ed., pp. 3-36, Pergamon, New York (1960).

27. V. S. R. Gudimetla and J. F. Holmes, "Probability density function of the intensity for a laser-generated speckle field after propagation through the turbulent atmosphere," J. Opt. Soc. Am. 72, 1213-1218 (1982).

28. J. W. Goodman, "Statistical properties of laser speckle patterns,' Chap. 2 in Laser Speckle and Related Phenomena, J. C. Dainty, Ed., Springer-Verlag, New York (1975).

29. L. C. Andrews, Special Functions of Mathematics for Engineers, 2nd ed., SPIE Optical Engineering Press, Bellingham, WA; Oxford University Press, Oxford (1998)[formerly published as 2nd ed. by McGraw-Hill, New York (1992)]. 